LBNL-41395

\title{
Medium Modified Cross Sections, Temperature and Finite Momentum Effects for Antikaon Production in Heavy-Ion Collisions*
}

\author{
Jürgen Schaffner-Bielich \\ RIKEN BNL Research Center, Brookhaven National Laboratory, \\ Upton, New York 11973, USA \\ Volker Koch \\ Nuclear Science Division, Lawrence Berkeley National Laboratory, \\ University of California, Berkeley, California 94720, USA \\ Martin Effenberger \\ Institut für Theoretische Physik, Universität Giessen, D-35392 Giessen, Germany
}

(September 4, 2018)

\begin{abstract}
The medium modifications of antikaons in dense matter are studied in a coupled channel calculation for scenarios more closely related to the environment encountered in heavy-ion collisions. We find that the optical potential of the antikaons turns repulsive or is drastically reduced for finite momenta or finite temperature. Hence, the antikaon mass does not decrease substantially in heavy-ion collisions to provide an explanation for the observed antikaon production rates at threshold. We demonstrate that the in-medium production cross section of antikaons via pions and $\Sigma$ hyperons is remarkably enhanced up to an order of magnitude. The effect of in medium correction on the resulting kaon spectra is studied within a transport model. We find that both in medium correction lead to about the same enhancement of the spectrum. However, once the temperature dependence is taken into account no enhance-
\end{abstract}

\footnotetext{
*This work was supported by a NATO collaborative research grant number 970102. This work was also partly supported by BMBF, DFG, GSI, Alexander von Humboldt-Stiftung, and by the Director, Office of Energy Research, Office of High Energy and Nuclear Physics, Nuclear Physics Division of the U.S. Department of Energy under Contract No. DE-AC03-76SF00098.
} 
ment is found.

13.75.Jz, 25.75.Dw, 25.80.-e, 24.10.Eq

Typeset using REVTEX 


\section{INTRODUCTION}

Antikaons have been proposed as a tool to probe in-medium effects in heavy-ion collisions. The work of Nelson and Kaplan [1] speculating about a strange condensate realignment in relativistic heavy ion collision by antikaons initiated numerous work in this field. Indeed, coupled channel calculations for antikaons in matter [2] demonstrate that the $\mathrm{K}^{-}$feels an attractive potential in dense matter, which was confirmed by [3, 4] and [5]. The main effect stems from the $\Lambda(1405)$ resonance which is shifted upwards at finite density (see 60 and references therein). Also, data from kaonic atoms indicates that the $\mathrm{K}^{-}$feels a sizable attraction inside the nucleus [7]. More theoretical foundation for a reduced effective antikaon mass has been also given using chiral perturbation theory [8,9] and mean-field models [10 12].

This has triggered a considerable interest in the production of $\mathrm{K}^{-}$in heavy-ion collisions at threshold. Indeed, an enhanced production of $\mathrm{K}^{-}$has been observed at GSI [13, 14]. This phenomenon has been attributed to a reduction of the antikaon effective mass in the dense medium [15 18].

An attractive potential for antikaons has been extracted from kaonic atoms at zero momentum [7]. However, heavy-ion collisions probe the optical potential of the antikaon at a finite momentum. Assuming a temperature of $T=80 \mathrm{MeV}$ [14, the antikaon has an average momentum of more than $300 \mathrm{MeV}$ with respect to the matter restframe. Furthermore, all experimental data available so far cover only momenta above $\sim 300 \mathrm{MeV}$ with respect to the matter rest frame. Calculations have so far assumed that the attractive potential for the kaons i.e. their change in the mass is independent of momentum. This constitutes a considerable extrapolation. To which extend this is justified will be addressed in the present paper.

Based on a dynamical model for the $\mathrm{K}^{-}$-nucleon interaction [2], we will argue that the antikaon optical potential in the momentum range relevant for heavy-ion collisions is likely to be small if not repulsive. Only for the smallest momenta, which are considerably below the range covered by experiment, we find attraction which is compatible with fits to kaonic atoms. Including the selfenergy of the kaon in a selfconsistent calculation we find that the optical potential at finite momentum is attractive but considerably reduced. Thus it is appears unlikely, that the reduction of the antikaon mass is the underlying in-medium effect responsible for the enhanced $\mathrm{K}^{-}$production rate. Instead, we find that the in-medium cross section for producing antikaons via $\Sigma$ hyperons might be the relevant mechanism for the observed enhancement. There are indications from stopped $\mathrm{K}^{-}$data on nuclei that there is indeed a change of the branching ratios in the nuclear medium 19 supporting that picture. Nevertheless, we also demonstrate that temperature effects are crucially important in the sense that they can wash out any in-medium effect.

The paper is organized as follows: we introduce the coupled channel approach in Sect. III and calculate medium effects for the kaon optical potential and cross section in Sect. III. Effects of the nucleon and kaon selfenergy are studied in Sect. IV. The in-medium effects for antikaons are included in a BUU transport model in Sect. $\nabla$ and contrasted with experimental data. 


\section{COUPLED CHANNEL MODEL FOR ANTIKAONS}

In the following we adopt the nonperturbative coupled channel approach of [2, 19] starting from the coupled channel Schrödinger equation,

$$
\nabla^{2} \psi_{i}(r)+k_{i}^{2} \psi_{i}(r)-2 \mu_{i} \int V_{i, j}\left(r, r^{\prime}\right) \psi_{j}\left(r^{\prime}\right) d^{3} r^{\prime}=0
$$

where $\psi_{i}(r)$ represents the relative wave function, $\mu_{i}$ is the reduced mass, and $k_{i}$ is the momentum in the center of mass system for the corresponding channel. For a separable potential of the form

$$
V_{i, j}\left(k, k^{\prime}\right)=g^{2} C_{i, j} v_{i}(k) v_{j}\left(k^{\prime}\right)=\frac{g^{2}}{\Lambda^{2}} C_{i, j} \Theta\left(\Lambda^{2}-k^{2}\right) \Theta\left(\Lambda^{2}-k^{\prime 2}\right)
$$

the scattering $T$-matrix simplifies to

$$
T_{i, j}\left(k, k^{\prime}, E\right)=g^{2} v_{i}(k) v_{j}\left(k^{\prime}\right)\left[(1-C \cdot G(E))^{-1} \cdot C\right]_{i, j} .
$$

The propagator matrix $G$ is diagonal and in the vacuum given by

$$
\begin{aligned}
g_{i}(E) & =g^{2} \int \frac{d^{3} p}{(2 \pi)^{3}} \frac{v_{i}^{2}(p)}{E-m_{i}-M_{i}-p^{2} /\left(2 \mu_{i}\right)} \\
& =\frac{1}{2 \pi^{2}} \frac{g^{2}}{\Lambda^{2}} \int_{0}^{\Lambda} \frac{p^{2} d p}{E-m_{i}-M_{i}-p^{2} /\left(2 \mu_{i}\right)} .
\end{aligned}
$$

The coupling matrix $C_{i j}$ is chosen to be of the standard $S U(3)$ flavor symmetric form. In addition to the previous approaches of [2,19], we include also the isospin one channels explicitly. For isospin zero, there are two channels

$$
C_{i j}^{I=0}=\left(\begin{array}{cc}
\bar{K} N & \pi \Sigma \\
-\frac{3}{2} & -\frac{\sqrt{6}}{4} \\
-\frac{\sqrt{6}}{4} & -2
\end{array}\right) \quad \begin{gathered}
\bar{K} N \\
\pi \Sigma
\end{gathered}
$$

whereas for the isospin one part, one has three channels which are coupled as

$$
C_{i j}^{I=1}=\left(\begin{array}{ccc}
\bar{K} N & \pi \Sigma & \pi \Lambda \\
-\frac{1}{2} & -\frac{1}{2} & -\frac{\sqrt{6}}{4} \\
-\frac{1}{2} & -1 & 0 \\
-\frac{\sqrt{6}}{4} & 0 & 0
\end{array}\right) \bar{K} N
$$

The coupled channel calculation is performed for the two isospin channels separately. The scattering amplitude in a given isospin channel is then

$$
f_{i, j}^{I}\left(k, k^{\prime}\right)=-\frac{\sqrt{\mu_{i} \mu_{j}}}{2 \pi} T_{i, j}^{I}\left(k, k^{\prime}\right)
$$


The cross section of a given channel is given by

$$
\sigma_{i j}=4 \pi \frac{k^{\prime}}{k}\left|f_{i j}\left(k, k^{\prime}\right)\right|^{2}
$$

and is constructed out of the two isospin components of the $T$ matrix.

The two parameters $\Lambda$ and $g$ can be fixed either by the scattering amplitude as extracted by Martin [20 or by the threshold ratios as in [21]

$$
\begin{aligned}
\gamma & =\frac{\Gamma\left(K^{-} p \rightarrow \pi^{+} \Sigma^{-}\right)}{\Gamma\left(K^{-} p \rightarrow \pi^{-} \Sigma^{+}\right)}=2.36 \pm 0.04 \\
R_{c} & =\frac{\Gamma\left(K^{-} p \rightarrow \text { charged particles }\right)}{\Gamma\left(K^{-} p \rightarrow \text { all }\right)}=0.664 \pm 0.011 \\
R_{n} & =\frac{\Gamma\left(K^{-} p \rightarrow \pi^{0} \Lambda\right)}{\Gamma\left(K^{-} p \rightarrow \text { all neutral states }\right)}=0.189 \pm 0.015
\end{aligned}
$$

We choose the former way as in [2] and take over the parameters $\Lambda=0.78 \mathrm{GeV}$ and $g^{2}=17.9$. We note in passing, that this choice of parameters gives the threshold ratios $\gamma=4.11, R_{c}=0.627$, and $R_{n}=0.234$. With a slight change of the coupling constant, i.e. for $g^{2}=16.8$, one gets the values $\gamma=2.37, R_{c}=0.644$, and $R_{n}=0.121$ but the pole position of the $\Lambda(1405)$ is shifted then towards $1420 \mathrm{MeV}$. This is consistent with the findings in [21], that one can either describe the scattering amplitude or the threshold ratios with a slight change of the coupling constant. We have verified that our results presented in the following are not sensitive to these small changes in the coupling constant $g$.

Nuclear medium effects can be easily included. Pauli blocking effects forbid intermediate nucleon states with a momentum less than the Fermi momentum. Hence, the propagator in the $\bar{K} N$ channel is modified in the medium to

$$
g_{1}\left(E, k_{f}\right)=\frac{g^{2}}{\Lambda^{2}} \int_{0}^{\Lambda} \frac{d^{3} p}{(2 \pi)^{3}} \frac{\Theta\left(k_{f}-\left|\vec{p}+M_{N} \vec{v}_{\Lambda}\right|\right)}{E-m_{K}-M_{N}-p^{2} /\left(2 \mu_{K p}\right)}
$$

in both isospin channels. Here, effects from a moving $\Lambda(1405)$ resonance is taken into account by the term proportional to the velocity of the resonance $v_{\Lambda}$ [19]. For a kaon with finite momentum with respect to the nuclear matter rest frame the intermediate nucleon propagator will be shifted in momentum space. Hence, the Pauli blocking effect will be reduced in that case and the effects stemming from the $\Lambda(1405)$ resonance are moderated.

The possibility for large range terms in the kaon-nucleon interaction, which might be important in the medium, has been raised in [9]. Fits to the $\mathrm{K}^{-}-\mathrm{N}$ scattering data using a coupled channel formalism find a good description without a range term [22,23]. Also in the model presented above (without range terms) one gets a good agreement with the scattering amplitude far below threshold [2]. Hence, we will not consider range terms in the following.

\section{OPTICAL POTENTIAL AND IN-MEDIUM CROSS SECTIONS}

Antikaons produced in heavy-ion collisions will change their properties due to the surrounding nucleons. The antikaons produced in the first collisions will suffer rescattering 
processes from the in-streaming nucleons. The surrounding matter is not like bulk matter, it is more like a streaming matter with a high momentum. Therefore, the optical potential relevant for the production of kaons even at subthreshold energies has to be considered at a finite momentum in the range of $p \approx 250 \mathrm{MeV}$ relative to the matter rest frame. In the following we denote this shift in momentum space of the colliding antikaon-nucleon pair (with a given colliding energy $\sqrt{s}$ ) with respect to the rest frame of the nuclear bulk matter in short form as the relative momentum. Figure 1 shows the optical potential of the kaon as defined as

$$
U_{\text {opt }}=-\frac{2 \pi}{\mu_{K p}} \Re\left(\frac{1}{4} f_{11}^{I=0}+\frac{3}{4} f_{11}^{I=1}\right) \rho
$$

where we ignore recoil corrections. They are small [2] and not relevant for the implementation into cascade models. For low relative momenta, the optical potential is getting more and more attractive as the density increases. This changes remarkably at higher relative momentum. The optical potential changes sign and gets repulsive again. Especially for moderate densities, at normal nuclear density $\rho_{0}$ or less, the change happens at relative momenta not far from the Fermi momentum of the nucleons. For higher relative momenta, the optical potential stays repulsive. Hence, antikaon production is less favored at high relative momenta, on the contrary it is even slightly suppressed in the medium. Pauli blocking effects of the propagator in the $\bar{K} N$ channel, eq. (13), are responsible for switching the sign of the optical potential from repulsion at low density to attraction at high density (this is due to the $\Lambda(1405)$, see [2]). This mechanism is not working at high relative momenta (comparable to the Fermi momenta of the nucleons), therefore resulting in a repulsive optical potential for the antikaons. For a higher density of $2 \rho_{0}$ and more the $\Lambda(1405)$ melts in the medium and an attractive potential is seen even for large relative momenta. Therefore, one has to focus either on high density or on low to moderate density with small relative momentum to see the in-medium reduction of the antikaon mass in matter. Inclusive observables in heavy-ion collisions are likely to probe regions of phase space where the antikaon feels both attractive and repulsive potentials so that in-medium effects are washed out. We will discuss these effects by making a detailed comparison with experiment in section $\square$.

In the intermediate and final stage of the heavy-ion collision, the nuclear matter will be heated up. Typical slope parameters are in the range of $T=90 \mathrm{MeV}$ for the kaons at a bombarding energy of $1.8 \mathrm{AGeV}$ [13]. Temperature effects can be implemented in our approach by replacing the $\Theta$ function in the propagator (13) with a Fermi-Distribution:

$$
g_{1}\left(E, k_{f}\right)=\frac{g^{2}}{\Lambda^{2}} \int_{0}^{\Lambda} \frac{d^{3} p}{(2 \pi)^{3}} \frac{\left[\mathrm{e}^{\left(p^{2} /\left(2 M_{N}\right)-\nu\right) / T}+1\right]^{-1}}{E-m_{K}-M_{N}-p^{2} /\left(2 \mu_{K p}\right)},
$$

where $\nu$ is the chemical potential which is fixed for a given density $\rho$. Again, Pauli blocking effects will be weakened by a thermal smearing of the Fermi sphere. Indeed, as seen in Fig. 2, the optical potential for antikaons changes considerably for a finite temperature. At a temperature of $T=90 \mathrm{MeV}$, the optical potential has turned from attraction to repulsion for all cases shown up to a density of $\rho=2 \rho_{0}$. The temperature at which the change of signs happens is rather low, especially for moderate densities. At normal nuclear density, this critical temperature is around $T=30 \mathrm{MeV}$. 
Usually, the optical potential for $T=0 \mathrm{MeV}$ is used for describing the enhanced production of antikaons in heavy-ion collisions. The optical potential is repulsive at very low densities up to $\rho \approx 0.2 \rho_{0}$ due to the $\Lambda(1405)$ resonance. As the resonance mass is shifted up in dense matter (see [6]), the optical potential of the antikaons turns attractive for higher density. The switch in the sign of the optical potential from attraction to repulsion happens to occur at a much higher density, if the surrounding matter is moderately heated up. For a temperature of $T=90 \mathrm{MeV}$, this turning point is already shifted beyond $2 \rho_{0}$.

While the optical potential is essentially zero for momenta relevant for heavy ion collisions, we find a considerable increase of the production cross section $\pi+\Sigma \rightarrow K^{-}+N$. Figure 3 shows this enhancement factor which we define as the ratio of the cross section at normal nuclear density and the vacuum cross section $\sigma\left(\rho=\rho_{0}\right) / \sigma_{0}$ for the elastic reaction $K^{-} p \rightarrow K^{-} p$ and the charge exchange reaction $K^{-} p \rightarrow K^{0} n$. Both cross sections are enhanced tremendously. The elastic cross section is four times bigger already at threshold and increases up to a factor of 27 around an antikaon momentum close to the Fermi momentum of the nucleons. The charge exchange reaction sets in at a finite antikaon $\left(K^{-}\right)$momentum due to the mass difference of the charged and neutral antikaon. This cross section is even more enhanced in the medium than the elastic cross section signaling significant rescattering effects for the production of $K^{-}$in the medium.

Via detailed balance and by using eq. (9) we calculate the production cross section of antikaons in the medium by $\pi Y \rightarrow K^{-} p$. We expect that especially the channels involving $\Sigma$ hyperons will be enhanced in the medium as this channel enters into the isospin zero coupled channel. The main medium effects are resulting from the dynamics of the $\Lambda(1405)$ which changes the isospin zero part of the coupled channel calculation. The channel $\pi \Lambda \rightarrow K^{-} p$, which is isospin one, is then not changed significantly as evident from Fig. 4. We note that the antikaon production cross section $\pi \Sigma \rightarrow K^{-} p$ has increased by a large factor in matter. Especially, the reaction $\pi^{-} \Sigma^{+} \rightarrow K^{-} p$ is favored in dense matter compared to the other reactions at threshold. This results in a change of the branching ratio for nuclear targets, as discussed in [19]. We point out, that the present experimental data seems to support this medium effect for the reaction $\left(K^{-}, K^{+}\right.$) (see [19]). For higher antikaon momentum around $250 \mathrm{MeV}$, all three $\Sigma$ channels are enhanced by a factor of $20-30$.

The results presented here are not an artifact of our model used. One could argue that a chiral nonperturbative approach might alter the picture. Also the $\eta Y$ channel contributions have to be taken into account, so that the matrices (6) and (7) have to extended correspondingly. Moreover, especially concerning the pion dynamics, relativistic effects can be important. We have checked for all of these effects by using the recent chiral nonperturbative approach of Oset and Ramos [23] which uses relativistic propagators and includes effects from the $\eta Y$ channels (for a recent extension of their model see [24]). The propagators are solved numerically by using a Cauchy integrator. The separable potential originating from the lowest order chiral Lagrangian term are now of the form

$$
V_{i j}=-C_{i j} \frac{1}{4 f^{2}}\left(k^{0}+k^{\prime 0}\right)
$$

and momentum dependent. The calculation of the $T$-matrix in the medium follows essential along the lines as described in section [1]. The coupling matrix as well as the expression for the propagator and the cross section can be found in [23]. Note that this chiral approach 
provides a good description of the available vacuum scattering data. In the following, we take the parameters of [23], $f=1.15 f_{\pi}$ and a cutoff of $0.63 \mathrm{GeV}$. We remark on passing that the threshold ratios in the isospin basis seems to be off but can be adjusted to the experimental data by a small change of the parameters. For a coupling constant of $f=122.9 \mathrm{MeV}$ and a momentum cutoff of $\Lambda=0.8 \mathrm{GeV}$, we find the threshold ratios $\gamma=2.35, R_{c}=0.635$, and $R_{n}=0.178$ which are in agreement with the data except for $R_{c}$ which turns out to be a little bit too small. As found for the non-relativistic case before, the position of the $\Lambda(1405)$ is shifted up towards $1.42 \mathrm{GeV}$.

Medium effects are introduced in a similar fashion as discussed before. The resulting in-medium enhancement factor of the cross section is shown in Fig. 1 in comparison to the nonrelativistic case. The $\pi \Lambda$ channel is not as enhanced as the $\pi \Sigma$ channels, but shows a rise in the chiral approach compared to the nonrelativistic approach. The $\pi^{-} \Sigma^{+}$production channel is enhanced at threshold even for this approach. Also, all three $\pi \Sigma$ channels are drastically enhanced around an antikaon momentum of $250 \mathrm{MeV}$ by a factor between 20-40. These findings support the picture already seen in the nonrelativistic case.

Now, we turn to study effects of a finite relative momentum with respect to the matter rest frame and temperature in the nonrelativistic case. The enhancement factor for the production of $\mathrm{K}^{-}$are depicted in Fig. 5 at finite relative momentum and in Fig. 6 for finite temperature, respectively. Both plots show the average cross section for producing a $\mathrm{K}^{-}$via $\Sigma$ hyperons. Similar to the optical potential the effect decreases at finite relative momentum. However, the cross section is still enhanced by a factor three at threshold even for a relative momentum of $p=450 \mathrm{MeV}$, where the optical potential of the antikaon is already repulsive (see Fig. 1). The temperature dependence of the cross section is even stronger as anticipated from our discussion about temperature effects for the antikaon optical potential. The peak structure vanishes already at a temperature of $T=30 \mathrm{MeV}$. Still, an enhancement factor of 2-3 remains at threshold even for the highest temperature shown $(T=90 \mathrm{MeV})$ for which the optical potential for the antikaon is repulsive throughout the density region of interest (see Fig. 2).

\section{EFFECTS FROM THE SELFENERGY}

A point of criticism can be raised in connection with the general approach. If the $\Lambda(1405)$ as a resonance melts in hot and dense matter what should remain is the meanfield potential due to the underlying antikaon-nucleon potential. This can be tested by e.g. including an extra imaginary optical potential into the propagator which simulates collisions with surrounding nucleons. The potential is known from proton-Nucleus scattering data to be about $V_{N N} \approx-10 \mathrm{MeV}$ at $\rho_{0}$ (see e.g. [25]). Compared to the kaon optical potential inside the nucleus we expect a $10 \%$ effect if we include the nucleon optical potential in the propagator (13).

The enhancement seen in the cross section originates from the dynamics of the $\Lambda(1405)$ resonance. As we have demonstrated, it does not depend on the detail of the interaction. Nevertheless, as recently shown by Lutz [5], the $\Lambda(1405)$ does not move to higher energy, if the propagator is calculated selfconsistently by including the self-energy of the antikaons. The peak of the imaginary part of the isospin zero scattering amplitude is not shifted but gets broader in the medium. Hence, strength is still transported from the off-shell energy 
towards threshold energy as the peak broadens. Using the optical theorem, this means that the cross section will still be enhanced at threshold in the medium. The enhancement in the medium will be less pronounced as in our approach but we estimate from Fig. 4 of [5] that it will be still a factor of four or so enhanced compared to the vacuum.

Both selfenergies can be incorporated in our approach by modifying the propagator (13) to

$$
\begin{aligned}
g_{1}\left(E, k_{f}\right) & =\frac{g^{2}}{\Lambda^{2}} \int_{0}^{\Lambda} \frac{d^{3} p}{(2 \pi)^{3}} \\
& \times \frac{\Theta\left(k_{f}-\left|\vec{p}+M_{N} \vec{v}_{\Lambda}\right|\right)}{E-m_{K}-W_{K}\left(E-M_{N}-E_{N}(p), p_{C M}-p ; \rho\right)-M_{N}-i V_{N N}-p^{2} /\left(2 \mu_{K p}\right)}
\end{aligned}
$$

where $W_{K}$ stands for the optical potential of the kaon which has to be calculated selfconsistently. Note that the optical potential has now a real and an imaginary part, where the former part is defined by eq. (14) and the latter part correspondingly. The optical potential $W_{K}$ is calculated iteratively and then put into the propagator in the next step. This iteration scheme converges after 5-6 steps in agreement with the calculations in [5]. We denote the results of this approach in the following as the selfconsistent ones.

The effects of the nucleon and the antikaon optical potential on the real part of the antikaon optical potential are summarized in Fig. 7. Shown is the momentum dependence at normal nuclear density. Our previous result without the implementation of any selfenergy for the nucleon or the antikaon is plotted in solid. At zero momentum, the optical potential changes by $10 \%$, as expected, when including the nucleon optical potential. The selfconsistent approach reduces the optical potential remarkably to $U_{K}\left(\rho_{0}\right)=-32 \mathrm{MeV}$ in stark contrast to the standard values deduced from Kaonic atoms [6]. On the other hand, the momentum dependence of the selfconsistently calculated optical potential is just flat. That means, that the optical potential does not turn repulsive anymore as it happens for the calculation with the free propagator. The nucleon optical potential also changes the momentum dependence considerably, so that the potential decreases but is still attractive at high momenta. This trend continues when applying values of $V_{N N}=-30,-50 \mathrm{MeV}$ : the optical potential saturates at $-60 \mathrm{MeV}$ at zero relative momentum and at $-40 \mathrm{MeV}$ at high relative momentum. Note, that for all cases considered here, the optical potential is still substantially weakened at high momenta (or even over the whole momentum range shown). We have also checked for a repulsive optical potential of the $\Sigma$ [26] of $U_{\Sigma}=+30 \mathrm{MeV}$ at $\rho_{0}$. Choosing in addition $U_{\pi}=+30 \mathrm{MeV}$ and $U_{\Lambda}=-30 \mathrm{MeV}$ at $\rho_{0}$, we find that the real part of the optical potential is reduced slightly by about $10 \%$. We also checked possible effects from an effective nucleon mass as done in [2] before. A nucleon mass shifted by $-50 \mathrm{MeV}$ at $\rho_{0}$ does not alter significantly the position and shape of the $\Lambda(1405)$ compared to the shifted threshold and hence can be safely neglected. This result is in line with refs. [3, 24] where binding energy effects for hyperons and nucleons have been found to be small, too.

We turn now again to the production cross section of $\mathrm{K}^{-}$via hyperons, i.e. the enhancement factors when including the nucleon optical potential (Fig. 8) and for the selfconsistent calculation (Fig. 9). The nucleon optical potential reduces the overall enhancement factor by about a factor of two. The shapes are essentially the same as for the free propagator, Fig. 3. For larger values of $V_{N N}=-30,-50 \mathrm{MeV}$ we find that the enhancement factors at the maximum are reduced down to $4-5$ and $2.5-3$, respectively. Effects of hyperon and pion 
optical potentials reduce the maximum enhancement factors down to $16-22$, i.e. a repulsive $\Sigma$ potential still results in a sizable enhancement of the $\pi \Sigma$ production cross section.

The selfconsistent case shows a quite different behavior. The $\pi^{0} \Sigma^{0}$ and the $\pi^{+} \Sigma^{-}$channels are enhanced at higher momenta of the outgoing antikaon while the $\pi^{-} \Sigma^{+}$channel increases especially at threshold up to a factor of 2.5. Compared to the calculation done in [5], a calculation using the chiral interaction terms provides a larger enhancement. We attribute this to the energy dependence of the chiral interaction term which will shift strength from lower to higher energies.

\section{KAON PRODUCTION IN HEAVY ION COLLISIONS WITH MODIFIED CROSS SECTIONS}

\section{A. Description of the model}

In order to explore the observable consequences of the results above we have performed calculations of $K^{-}$production in $\mathrm{Ni}+\mathrm{Ni}$ collisions at $1.8 \mathrm{AGeV}$ within a BUU transport model that has been quite successfully applied to particle production in heavy-ion collisions at SIS energies [27] as well as pion [28 and photon [29,30] induced reactions. For a detailed description of the underlying formalism and the the non-strange part of the model we refer to Refs. 27,29]. We note that for the calculations presented here the string fragmentation model FRITIOF [31] is not included.

\section{Strangeness production}

Our model contains the following strangeness production channels in baryon-baryon collisions:

$$
\begin{aligned}
& B B \rightarrow N \Lambda K \\
& B B \rightarrow N \Sigma K \\
& B B \rightarrow N N K \bar{K} .
\end{aligned}
$$

The cross section for $B B \rightarrow N Y K$ is parameterized in the following way:

$$
\sigma_{B B \rightarrow N Y K}=C \frac{1}{p_{i} \sqrt{s}}\left|\mathcal{M}_{Y}\right|^{2} 16(2 \pi)^{7} \Phi_{3}
$$

where $p_{i}$ is the cms momentum of the incoming particles and $\sqrt{s}$ is the total cms energy. $\Phi_{3}$ is the 3-body phase space as, for example given by Eq. (35.11) in Ref. [32]. For the isospin coefficient $C$ we adopt the one-pion-exchange model of Ref. [33]. We obtain the following expression of Clebsch-Gordan coefficients:

$$
C=\left|\left\langle\frac{1}{2} 1 m_{3} m_{\pi} \mid \frac{1}{2} 1 t_{1} m_{1}\right\rangle\right|^{2} \sum_{I} g_{Y}^{I}\left|\left\langle t_{2} 1 m_{2} m_{\pi} \mid t_{2} 1 I M\right\rangle\right|^{2}\left|\left\langle t_{Y} \frac{1}{2} m_{Y} m_{K} \mid t_{Y} \frac{1}{2} I M\right\rangle\right|^{2}+(1 \leftrightarrow 2),
$$


where $t_{1}$ and $t_{2}$ are the total isospins of the incoming baryons and $m_{1}$ and $m_{2}$ are their $z$-components. The $z$-component of the isospin of the outgoing nucleon is given by $m_{3}$. The coefficient $g_{Y}^{I}$ denotes the weight of the $Y K$-system with isospin $I$. For $\Lambda K$ we simply have $g_{\Lambda}^{1 / 2}=1$, for $\Sigma K$ we use $g_{\Sigma}^{1 / 2}=2 g_{\Sigma}^{3 / 2}=\frac{2}{3}$ in order to describe the experimental data as will be shown below.

The matrix element $\left|\mathcal{M}_{Y}\right|^{2}$ is parameterized as:

$$
\left|\mathcal{M}_{Y}\right|^{2}=a_{Y}\left(\frac{1}{s / \mathrm{GeV}^{2}-3.94}\right)^{1.781}
$$

with $a_{\Lambda}=59.3 \mathrm{mb}$ and $a_{\Sigma}=44.5 \mathrm{mb}$. In Fig. 10 we show that this gives a reasonable description of the experimental data for proton-proton collisions. Unfortunately there are presently no experimental data for proton-neutron collisions in the energy range of interest available. We note that the one-pion-exchange model gives:

$$
\sigma_{p n \rightarrow N \Lambda K}=5 \sigma_{p p \rightarrow p \Lambda K^{+}}
$$

because the model favors transitions with total isospin $I_{t o t}=0$. This might not be realistic. Under the assumption that the total matrix element does not depend on the total isospin one gets $\sigma_{p n}=\sigma_{p p}$ which is a factor of 5 smaller. For the process $B B \rightarrow N N K \bar{K}$ we use the parameterization given in 18 .

In the meson-baryon sector our model includes the following processes for strangeness production:

$$
\begin{aligned}
& \pi N \leftrightarrow \Lambda K \\
& \pi N \leftrightarrow \Sigma K \\
& \pi N \rightarrow N K \bar{K} \\
& \pi \Delta \leftrightarrow \Lambda K \\
& \pi \Delta \leftrightarrow \Sigma K \\
& \pi \Delta \rightarrow N K \bar{K} .
\end{aligned}
$$

The cross sections for $\pi N \rightarrow Y K$ and $\pi \Delta \rightarrow Y K$ are adopted from Ref. [34]. For $\pi N \rightarrow N K \bar{K}$ we use the parameterization of Ref. [35]. For $\pi \Delta \rightarrow N K \bar{K}$ the same isospin averaged cross section is employed. The different isospin contributions are calculated using the Feynman diagram of Ref. [35] with a $\Delta$ instead of a nucleon. The $\pi N$ and $\pi \Delta$ cross sections described here are also used for $\pi N^{\star}$ and $\pi \Delta^{\star}$ scattering.

In addition, our model includes the process:

$$
\pi \pi \rightarrow K \bar{K}
$$

where we adopt the isospin averaged cross section from [18] and calculate the isospin coefficients under the assumption that the matrix element does not depend on the total isospin and the different contributions add up incoherently. 


\section{Kaon-nucleon interaction}

In the $S=1$ channel our model contains the processes:

$$
\begin{aligned}
& K N \rightarrow K N \\
& K N \rightarrow K N \pi,
\end{aligned}
$$

where the used cross sections are described in detail in Ref. [30].

In the $S=-1$ channel we usually take into account the hyperon resonances explicitly 30]. In the present work they are not explicitly propagated in order to allow for an easier implementation of the medium modifications described above. Without the explicit resonance propagation their contributions are added to the 'background' cross sections. The following processes are included:

$$
\begin{aligned}
& \bar{K} N \rightarrow \bar{K} N \\
& \bar{K} N \leftrightarrow \pi \Lambda \\
& \bar{K} N \leftrightarrow \pi \Sigma \\
& \bar{K} N \rightarrow \pi \pi \Lambda, \pi \pi \Sigma, \pi \bar{K} N .
\end{aligned}
$$

The used cross sections can be found in Ref. 30]. We have checked that an explicit inclusion of the hyperon resonances does not change our results. We have also verified that the vacuum cross sections from the coupled channel model described above agree very well with the cross sections that are normally used in our transport model. For the present work we always use for invariant energies $\sqrt{s}$ below $1.52 \mathrm{GeV}$ the cross sections from the coupled channel model, i.e. also for the calculations without medium modifications.

\section{B. Results}

First, we note that our model gives a reasonable description of the experimental data on pion production and nucleonic observables in $\mathrm{Ni}+\mathrm{Ni}$ collisions at $1.8 \mathrm{AGeV}$. In Fig. 11] we compare our results for the $K^{+}$spectrum with the experimental data from Ref. [13. One sees that our calculation (solid line) describes the experimental data reasonably well although we underestimate the spectrum by about 30\%. We also show the result of a calculation (dashed line) for which we did not take into account the experimental acceptance cut $\left(40^{\circ}<\theta_{l a b}<48^{\circ}\right)$ for the kaon. This curve agrees better with the data. Compared to the calculation with acceptance cut the slope of the spectrum is larger. At this place we should mention that the calculations in [17,18] obtain contributions to the spectrum for kaon center of mass energies below $50 \mathrm{MeV}$ for which the experimental acceptance vanishes.

Having shown that our model gives a fair description of total strangeness production we now turn to the investigation of observable consequences of the above described medium modifications for the antikaon. Since the medium effects for the cross sections as well as the potential are most pronounced at zero temperature and momentum we first present results for which we neglect the temperature and momentum dependence and take all quantities at $T=0$ and $p=0$ in order to explore the maximum possible effects. Note that in all 
calculations [17,18] the temperature and the momentum dependence of the optical potential for the antikaon have been neglected. In Fig. 12 we compare our calculations to the experimental data from Ref. [13]. Due to limited numerical statistics we did not apply the experimental angular acceptance cut $\left(40^{\circ}<\theta_{l a b}<48^{\circ}\right)$ to our calculations. Because of this we get contributions to the spectrum at low center of mass energies for which the experimental acceptance vanishes. We have checked that within the numerical fluctuations our results do not change when applying the cut although $30 \%$ effects like in the case of the $K^{+}$ can not be excluded.

From Fig. 12 one sees that the medium modification of the cross sections (dashed line) gives an enhancement of low energy $K^{-}$by about a factor of 2 compared to the calculation without medium modifications (solid line) but only a small effect for kinetic energies above $200 \mathrm{MeV}$. As stated above, the density dependent cross sections for $\pi Y$ and $\bar{K} N$ collisions from the coupled channel model has been implemented for invariant energies below $1.52 \mathrm{GeV}$ (solid lines in Figs. 5 and 6). Above that the usual vacuum cross sections have been used. The effect of the potential alone (dotted line in Fig. 12) is quite similar. A simultaneous application of modified cross sections and potential (dash-dotted line) leads to a result which gives an enhancement of low energy antikaons by about a factor of three and a rather well description of the experimental data except for the two lowest data points. One should note that the modification of the $\pi Y \leftrightarrow \bar{K} N$ cross sections also gives an enhanced $\bar{K}$ absorption. In Fig. 13 we therefore show the number of $\pi \Sigma \rightarrow \bar{K} N$ (dashed lines) and $\bar{K} N \rightarrow \pi Y$ (solid lines) collisions for the different scenarios. One sees that the modification of the cross sections enhances the $\bar{K}$ production in $\pi \Sigma$ collisions for invariant energies below $1.52 \mathrm{GeV}$ by about a factor of 3 . An inclusion of the potential leads to an increase of $\bar{K} N \rightarrow \pi Y$ collisions by about a factor of two since more antikaons are overall produced, especially in baryon-baryon and pion-baryon collisions, while the $\bar{K}$-production in $\pi \Sigma$-collisions is only slightly enhanced. Due to the attractive $\bar{K}$-potential the $\sqrt{s}$-distributions are shifted to lower energies. In the calculation with modified cross sections and potential the antikaon production in $\pi \Sigma$-collisions below $1.52 \mathrm{GeV}$ is increased by more than a factor 5 compared to the case without any medium modifications. However, also the $\bar{K}$ absorption is substantially increased.

In Fig. 14 we present the results of calculations for which the momentum or temperature dependence in addition to the density dependence of the cross sections or potential were taken into account. Now the medium effects on the $K^{-}$yield completely disappear because as discussed above - all modifications decrease with increasing momentum and temperature. Using the temperature dependent $K^{-}$potential from Fig. 2 we even get a slight reduction of the $K^{-}$yield because in the dynamical calculation of the heavy-ion collision the densitytemperature correlations are such that the $K^{-}$potential is almost always repulsive.

One should note that a transport theoretical calculation of the total $K^{-}$yield in heavyion collisions at SIS energies is encumbered with substantial uncertainties with respect to the input of unknown elementary cross sections, like e.g. $\Delta \Delta \rightarrow \bar{K} X$ or $\pi \Delta \rightarrow \bar{K} X$. In this context we again mention that even strangeness production in proton-neutron collisions at the relevant energies has not yet experimentally been measured. Therefore a determination of the $K^{-}$potential from the total yield appears to be difficult. An observable which might not be sensitive to these uncertainties is $K^{-}$flow. Therefore, we show in Fig. 15 the influence of the different in-medium scenarios on transverse $K^{-}$flow. The anti-flow which 
is present in the calculation without medium modifications (solid line) is hardly affected by the modification of the cross sections (dashed line). The same also holds if one includes the temperature or momentum dependence. The attractive $K^{-}$potential (at $p=T=0$ ) (dotted line) is able to compensate this shadowing effect and gives a positive $K^{-}$flow since now the antikaons follow the nucleons. An inclusion of the temperature dependence of the potential leads to a disappearance of this effect.

In Fig. 16 we compare our calculations to the experimental data of the FOPI collaboration [36] for the $K^{-} / K^{+}$ratio in ${ }^{96} \mathrm{Ru}+{ }^{96} \mathrm{Ru}$ collisions at $1.69 \mathrm{AGeV}$. Here we have applied the experimental acceptance cuts $\left(p_{t}>0.1 \mathrm{GeV}, 39^{\circ}<\theta_{\text {lab }}<135^{\circ}, p_{\text {lab }}<0.32 \mathrm{GeV}\right)$. One sees that already the calculation without medium modifications (solid line) gives a reasonable agreement with the experimental data although the calculated distribution seems to be slightly too flat. The calculation with modified cross sections (at $p=T=0$ ) gives an enhancement of the $K^{-} / K^{+}$ratio by about $50 \%$ in the measured phase space region and is also compatible with the experimental data. The attractive $\bar{K}$-potential $($ at $p=T=0)$ (dotted line) leads to an pronounced increase of the ratio towards mid-rapidity. However, within the measured rapidity range also this scenario is hardly distinguishable from the one without medium modifications. We note that the theoretical calculation in the lowest rapidity bin around $y^{0}=-1.4$ suffers from very low numerical statistics as the rapidity distributions are practically zero. The calculation with modified cross sections and potential (dash-dotted line) gives a rather flat distribution that overestimates the experimental data by about $50 \%$. An inclusion of the temperature dependence again gives a result which is even slightly below the calculation without medium modifications.

Our results can be compared with Refs. [17, 18, 37]. The calculation for $K^{-}$production in $\mathrm{Ni}+\mathrm{Ni}$ collisions at $1.8 \mathrm{AGeV}$ (Fig. 12) without employing medium modifications agrees rather well with the respective calculation in Ref. [17]. The calculation in Ref. [18] gives a result that is a factor of 2 smaller. The effect of the attractive $K^{-}$potential is weaker in our calculations than in [17,18]. While in our calculations the $K^{-}$yield at energies around $200 \mathrm{MeV}$ is only enhanced by about $50 \%$, the calculations in [17] and [18] obtain factors of 2 and 3 , respectively.

In the calculation of the $K^{-} / K^{+}$ratio in $\mathrm{Ru}+\mathrm{Ru}$ collisions at $1.69 \mathrm{AGeV}$ in Ref. [37] it appears that the experimental cuts have not been taken into account since there are contributions at midrapidity where the acceptance vanishes. Without in-medium modifications this calculation is far below the data points [36] while an attractive in-medium potential (at zero relative momentum) shifts the curve on top of the data points. We note that our calculation without experimental acceptance cut gives a ratio at midrapidity which is a factor of 3-4 larger than the calculation in Ref. [37].

\section{SUMMARY AND DISCUSSION}

We have studied the optical potential for antikaons in the nuclear medium for finite relative momenta and finite temperatures. We find that the optical potential changes remarkably and turns repulsive at a relative momentum comparable to the nucleon Fermi momentum and/or a finite temperature of a few tens of $\mathrm{MeV}$. The strong momentum dependence is reduced when the hadron selfenergies are taken into account selfconsistently but it results in a substantially shallower antikaon potential. Hence, antikaons produced in the 
central region of an heavy-ion collision are likely to feel a substantially less attractive if not repulsive potential. Therefore, it seems that contrary to previous assumptions the antikaon potential at zero temperature and momentum can not be reliably used to study in-medium effects in heavy-ion collisions.

On the other hand, we demonstrate that the cross section for producing antikaons via $\Sigma$ hyperons is considerably enhanced in the nuclear medium. This holds also for model calculations implementing chiral symmetry, relativistic propagators, optical potentials for nucleons or for a selfconsistent calculation of the antikaon selfenergy. The cross sections is always enhanced at threshold, especially for $\pi^{-} \Sigma^{+}$, even for finite relative momentum or finite temperature.

We implemented the in-medium effects for the antikaons within a BUU transport model. An enhancement of the antikaon production is seen for medium modified cross sections and a $K^{-}$potential which follows more closely the experimental data than the calculation without any of those in-medium effects. Including temperature effects, the in-medium enhancement vanishes and even turns to a suppression of $K^{-}$due to the repulsive potential felt by the $K^{-}$.

Let us finally stress again that the environment for antikaons is completely different in heavy-ion collisions compared to Kaonic atoms and neutron stars where one probes the potential at zero momentum and zero temperature. It appears that the enhanced subthreshold production of $K^{-}$is more subtle than hitherto assumed and deserves further study. Clearly, we do not have a consistent picture to explain the enhancement seen experimentally. Further work has to be done to address this problem as for example by studying in-medium effects using the chiral approach at finite momentum and temperature. Future experimental data triggering on low momenta and/or the high density stage of heavy-ion collisions might help to elucidate the possible in-medium effects for antikaons in matter.

\section{ACKNOWLEDGMENTS}

We are expressing our gratitudes to Gerry Brown for useful discussions and to Avraham Gal for critical comments. We are grateful to N. Herrmann for providing us with the experimental data from the FOPI-collaboration. J. S.-B. thanks the nuclear theory group at LBNL, where part of this work was completed, for their warm hospitality and thanks the Alexander von Humboldt-Stiftung for their support during that time. The work of M.E. and V.K. was supported by a NATO collaborative research grant number 970102. This work was supported partly by the BMBF, DFG, GSI, and the Director, Office of Energy Research, Office of High Energy and Nuclear Physics, Nuclear Physics Division of the U.S. Department of Energy under Contract No. DE-AC03-76SF00098. 


\section{REFERENCES}

[1] A. E. Nelson and D. B. Kaplan, Phys. Lett. B 192, 193 (1987).

[2] V. Koch, Phys. Lett. B 337, 7 (1994).

[3] T. Waas, N. Kaiser, and W. Weise, Phys. Lett. B 379, 34 (1996).

[4] T. Waas and W. Weise, Nucl. Phys. A625, 287 (1997).

[5] M. Lutz, Phys. Lett. B 426, 12 (1998).

[6] L. R. Staronski and S. Wycech, J. Phys. G 13, 1361 (1987).

[7] E. Friedmann, A. Gal, and C. J. Batty, Nucl. Phys. A579, 518 (1994).

[8] G. E. Brown, C.-H. Lee, M. Rho, and V. Thorsson, Nucl. Phys. A567, 937 (1994).

[9] C.-H. Lee, G. E. Brown, D.-P. Min, and M. Rho, Nucl. Phys. A585, 401 (1995).

[10] T. Maruyama, H. Fujii, T. Muto, and T. Tatsumi, Phys. Lett. B 337, 19 (1994).

[11] J. Schaffner, A. Gal, I. N. Mishustin, H. Stöcker, and W. Greiner, Phys. Lett. B 334, 268 (1994).

[12] J. Schaffner-Bielich, I. N. Mishustin, and J. Bondorf, Nucl. Phys. A625, 325 (1997).

[13] R. Barth et al. (KaoS Collaboration), Phys. Rev. Lett. 78, 4007 (1997).

[14] N. Herrmann et al. (FOPI Collaboration), Nucl. Phys. A610, 49c (1996).

[15] G. Q. Li, C. M. Ko, and X. S. Fang, Phys. Lett. B 329, 149 (1994).

[16] G. Q. Li and C. M. Ko, Phys. Rev. C 54, 2159 (1996).

[17] G. Q. Li, C.-H. Lee, and G. E. Brown, Phys. Rev. Lett. 79, 5214 (1997).

[18] W. Cassing, E. L. Bratkovskaya, U. Mosel, S. Teis, and A. Sibirtsev, Nucl. Phys. A614, 415 (1997).

[19] A. Ohnishi, Y. Nara, and V. Koch, Phys. Rev. C 56, 2767 (1997).

[20] A. D. Martin, Nucl. Phys. B 179, 33 (1981).

[21] P. B. Siegel and W. Weise, Phys. Rev. C 38, 2221 (1988).

[22] N. Kaiser, P. B. Siegel and W. Weise, Nucl. Phys. A594, 325 (1995).

[23] E. Oset and A. Ramos, Nucl. Phys. A635, 99 (1998).

[24] A. Ramos and E. Oset, nucl-th/9906016.

[25] L. G. Arnold, B. C. Clark, R. L. Mercer, and P. Schwandt, Phys. Rev. C 23, 1949 (1981).

[26] C. J. Batty, E. Friedman, and A. Gal, Prog. Theor. Phys. Suppl. 117, 227 (1994).

[27] S. Teis, W. Cassing, M. Effenberger, A. Hombach, U. Mosel, and G. Wolf, Z. Phys. A 356, 421 (1997).

[28] M. Effenberger, E. L. Bratkovskaya, W. Cassing, and U. Mosel, Phys. Rev. C 60, 27601 (1999).

[29] M. Effenberger, E. L. Bratkovskaya, and U. Mosel, Phys. Rev. C 60, 44614 (1999).

[30] M. Effenberger and U. Mosel, nucl-th/9908078.

[31] B. Anderson, G. Gustafson and Hong Pi, Z. Phys. C 57, 485 (1993).

[32] Particle Data Group, Eur. Phys. J. C 3, 1 (1998).

[33] J. Randrup and C.M. Ko, Nucl. Phys. A 343, 519 (1980).

[34] K. Tsushima, S.W. Huang, A. Faessler, Austral. J. Phys. 50, 35 (1997).

[35] A. Sibirtsev, W. Cassing, C.M. Ko, Z. Phys. A 358, 101 (1997).

[36] N. Herrmann, Prog. Part. Nucl. Phys. 42, 187 (1999).

[37] G. Q. Li and G. E. Brown, Phys. Rev. C 58, 1698 (1998).

[38] A. Baldini et al., it Landolt-Börnstein, Band 12, Springer-Verlag, Berlin, 1987. 


\section{FIGURES}

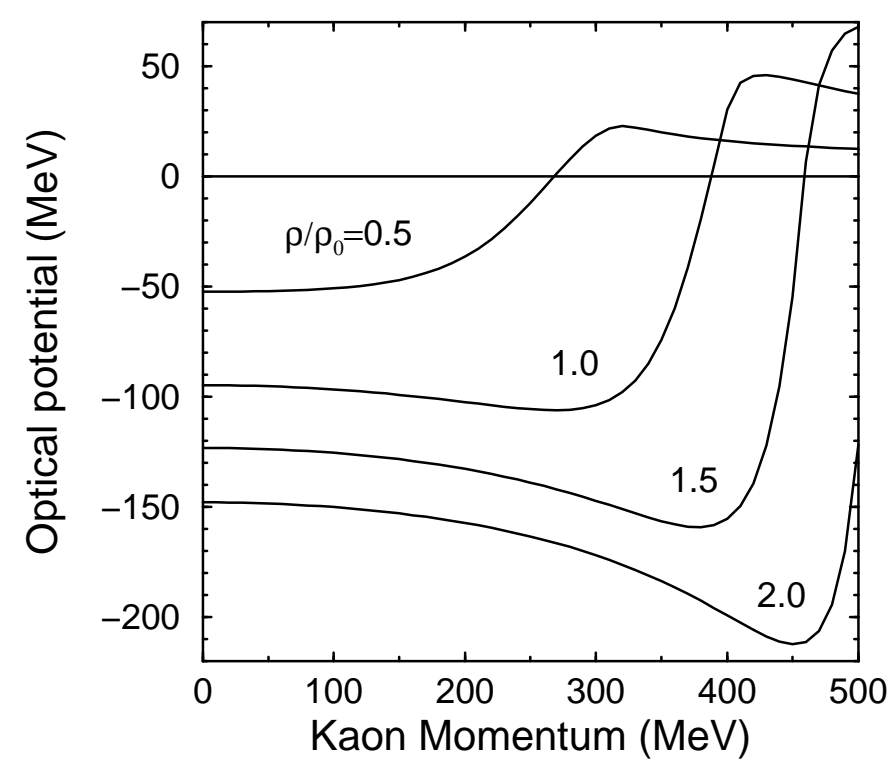

FIG. 1. The antikaon optical potential versus the antikaon relative momentum for different densities. The optical potential turns repulsive at a certain momentum especially for lower densities. At densities above $2 \rho_{0}$, the optical potential stays attractive even for high relative momenta.

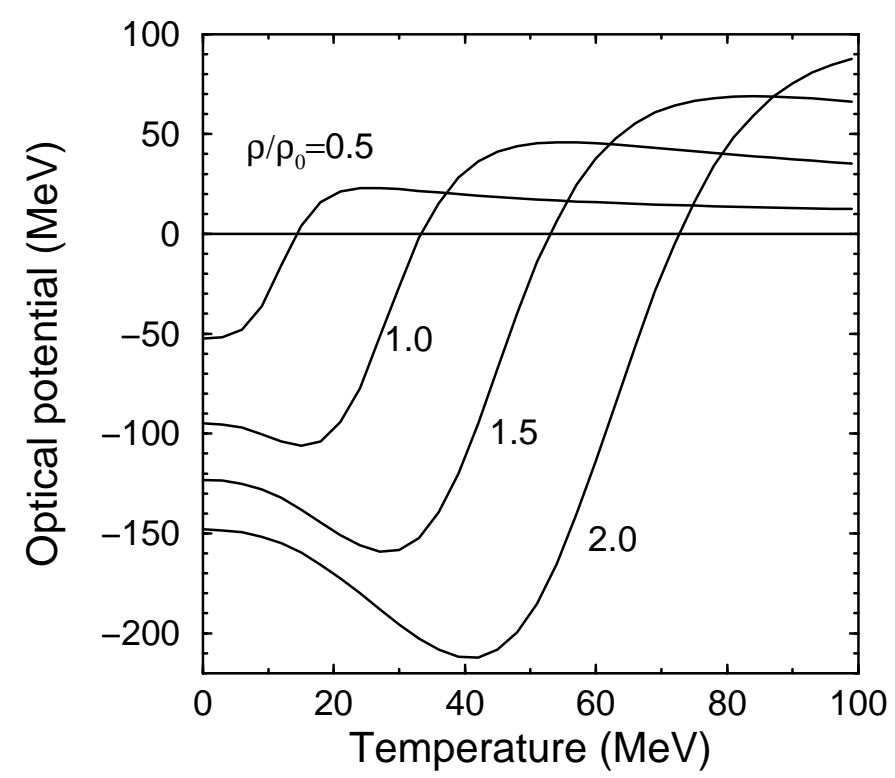

FIG. 2. The antikaon optical potential as a function of the temperature of the nucleons for different densities. The optical potential turns repulsive at a rather low temperature. 


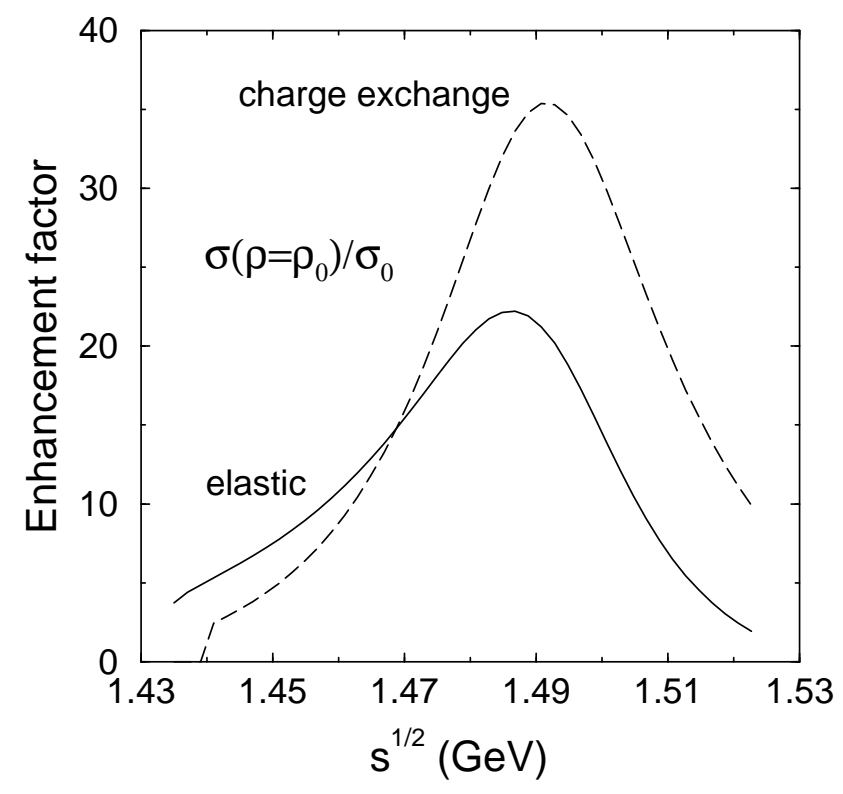

FIG. 3. The enhancement factor of the charge exchange reaction $K^{-}+p \rightarrow K^{0}+n$ and the elastic reaction at normal nuclear matter density.
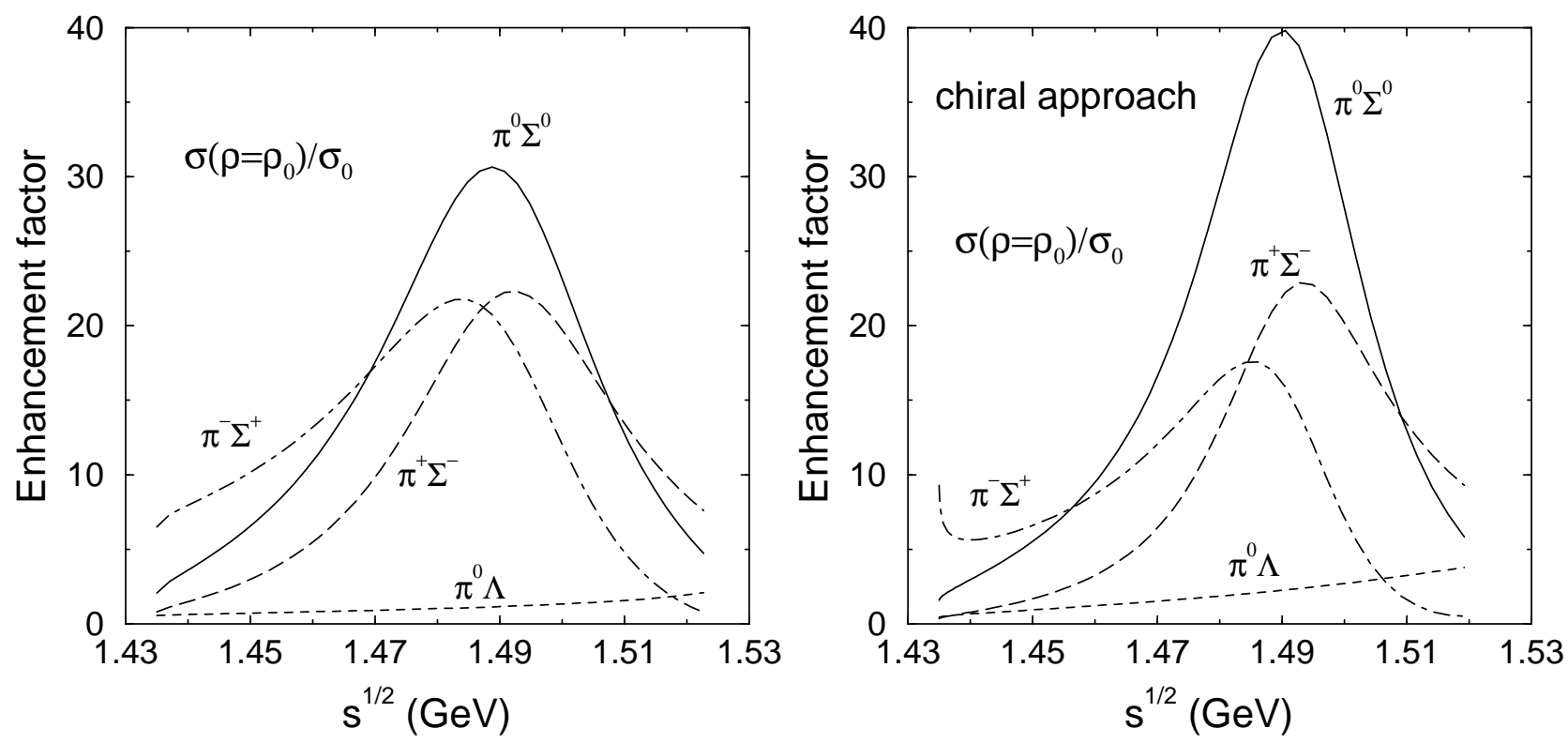

FIG. 4. Left figure: The enhancement factor of the antikaon production cross sections at normal nuclear density. The process $\pi+\Sigma \rightarrow p+K^{-}$is considerably enhanced in the medium.

Right figure: The same as left but using the nonperturbative chiral approach of [23] with relativistic propagators. 


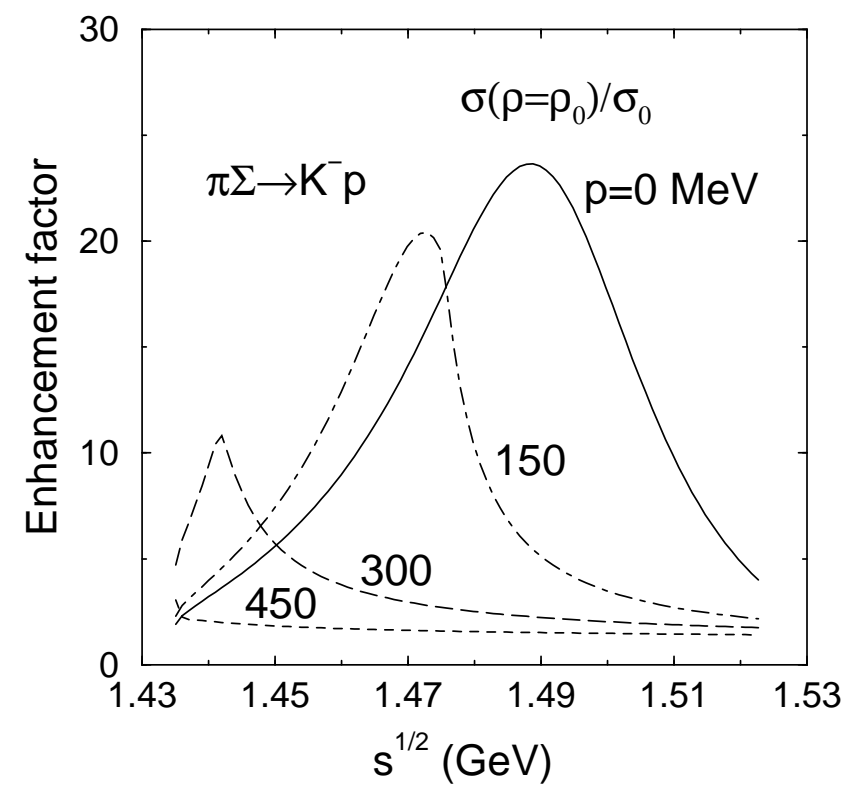

FIG. 5. The hyperon induced cross section for antikaon production at finite relative momenta with respect to the matter rest frame.

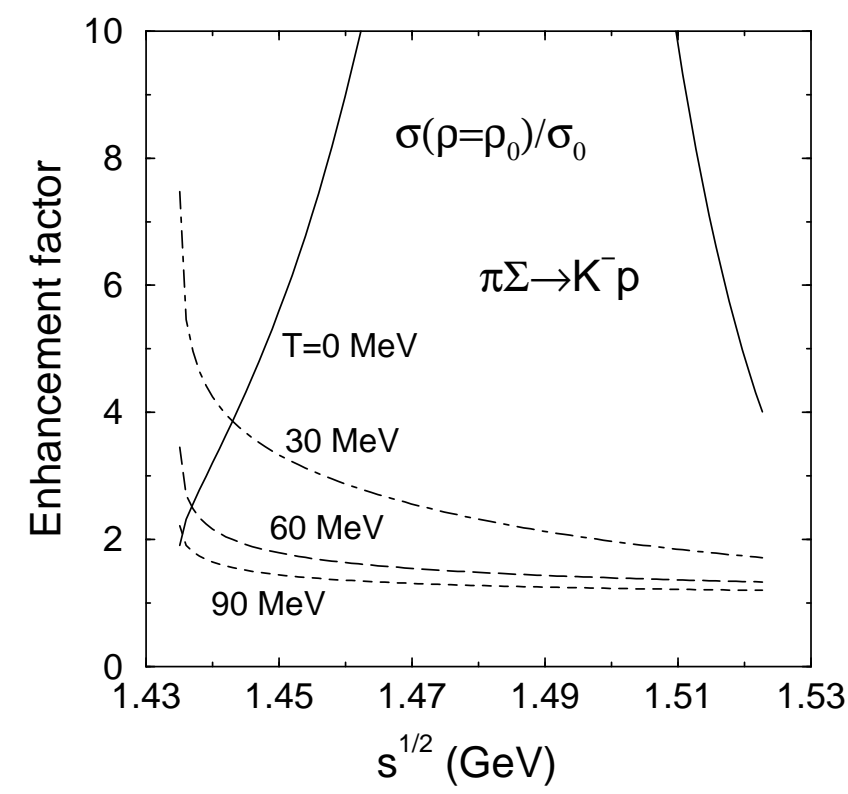

FIG. 6. The hyperon induced cross section for antikaon production at finite temperature. 


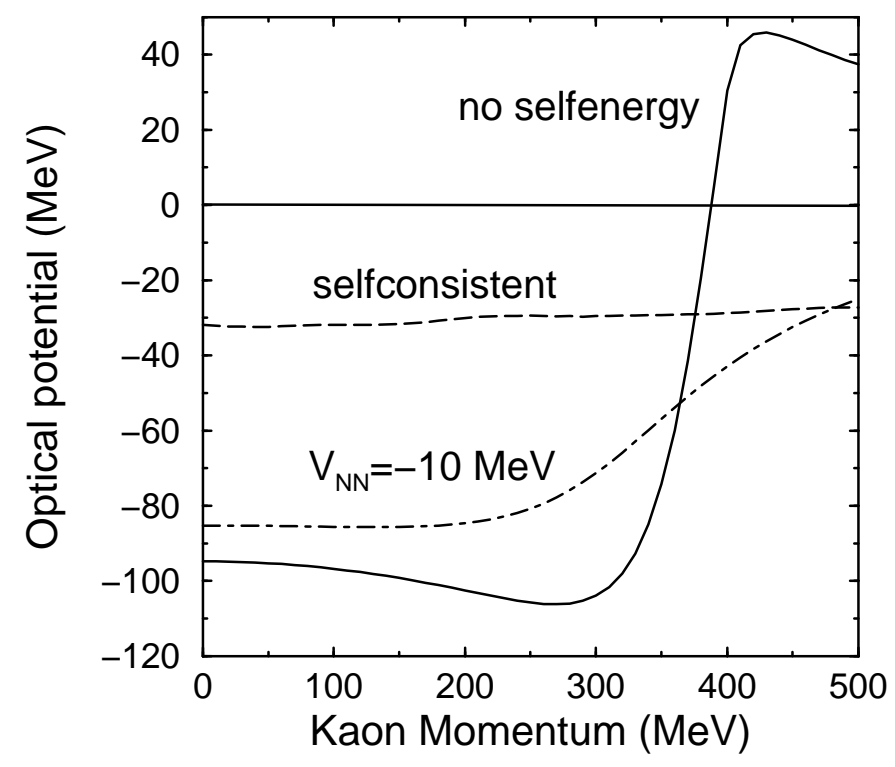

FIG. 7. The optical potential including the selfenergy for the nucleons (dash-dotted line) or antikaons (dashed line) as a function of the relative momentum with respect to the nuclear matter rest frame.

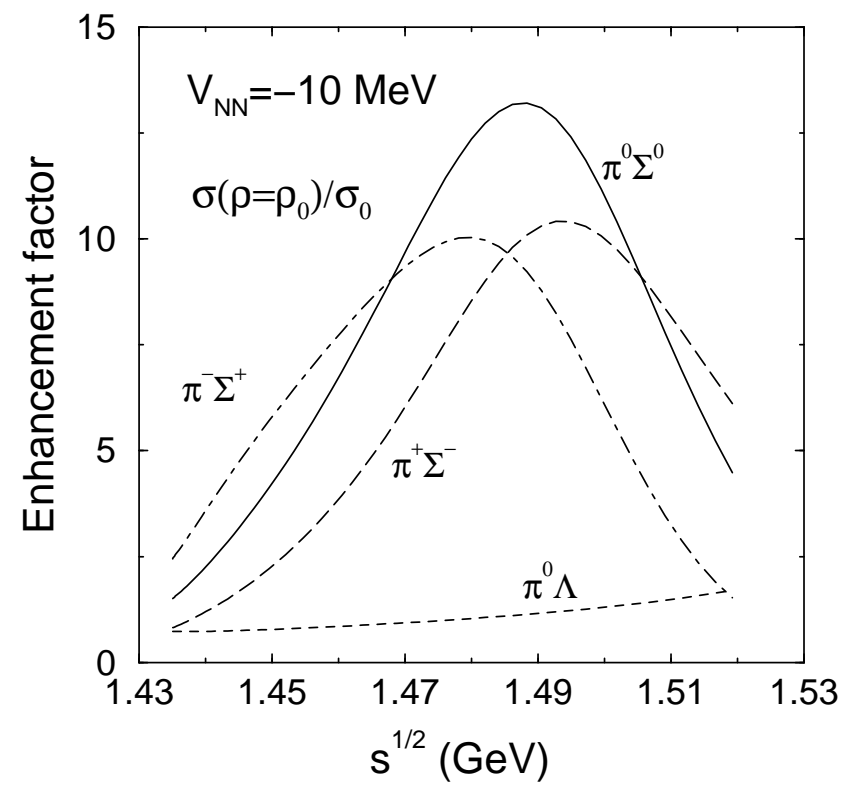

FIG. 8. The enhancement factor of the antikaon production cross sections at normal nuclear density including an imaginary potential for the nucleons. 


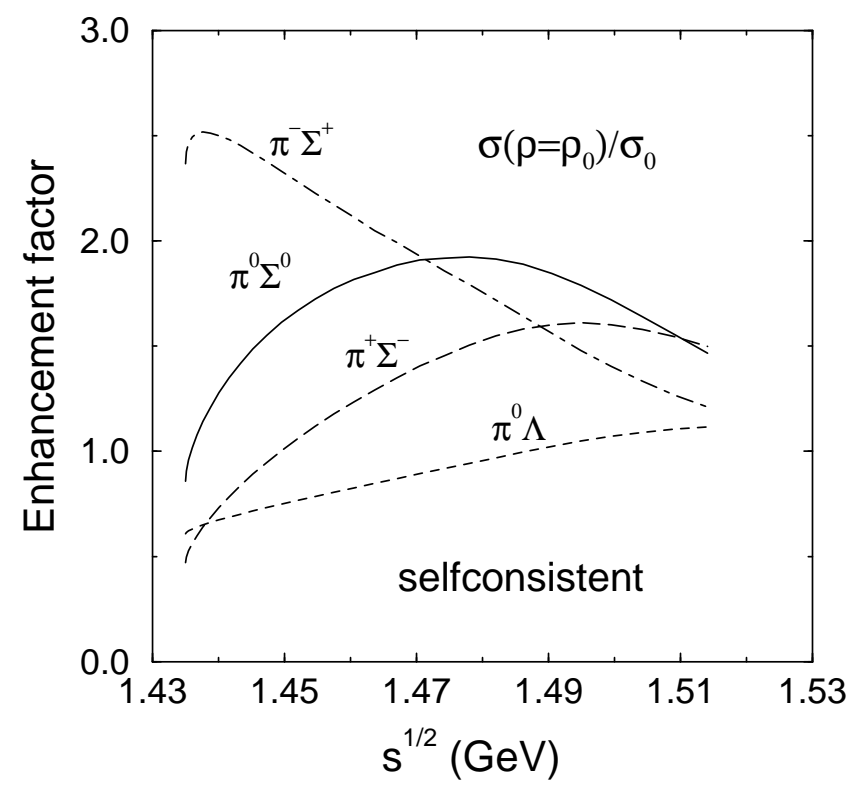

FIG. 9. The enhancement factor of the antikaon production cross sections at normal nuclear density for a selfconsistently calculated selfenergy of the antikaon.
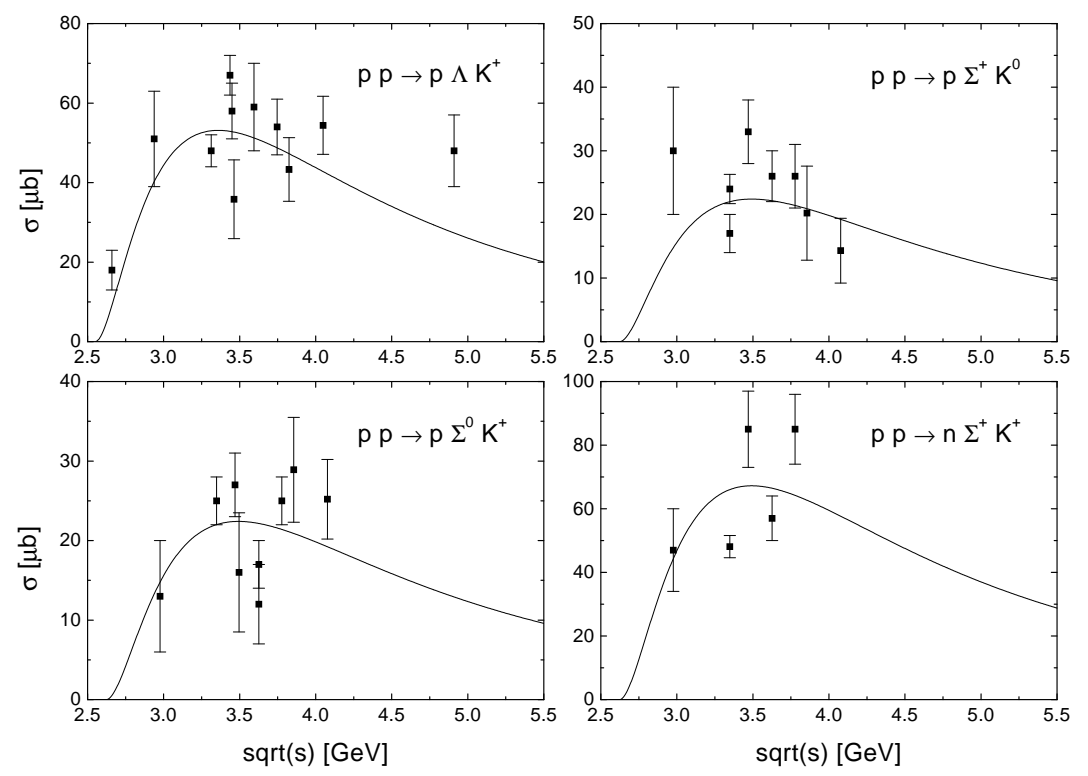

FIG. 10. Cross sections for $p p \rightarrow N Y K$. The experimental data are taken from Ref. [38]. 


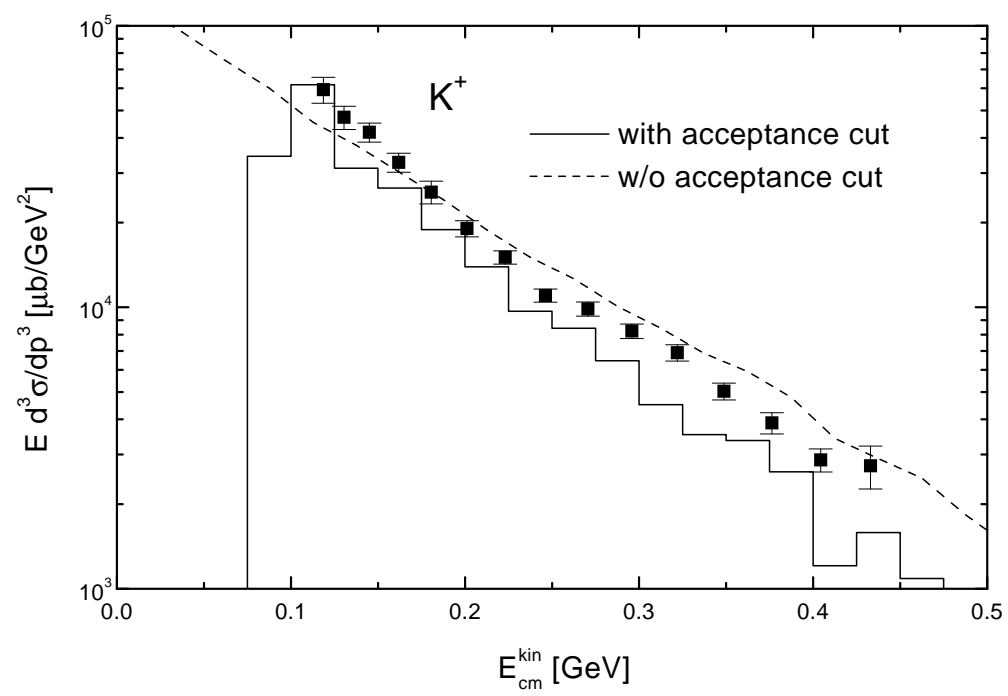

FIG. 11. Inclusive invariant $K^{+}$production cross section for $\mathrm{Ni}+\mathrm{Ni}$ collisions at $1.8 \mathrm{AGeV}$. For the dashed line the experimental acceptance cut $\left(40^{\circ}<\theta_{l a b}<48^{\circ}\right)$ was not taken into account. The experimental data are taken from Ref. 113.

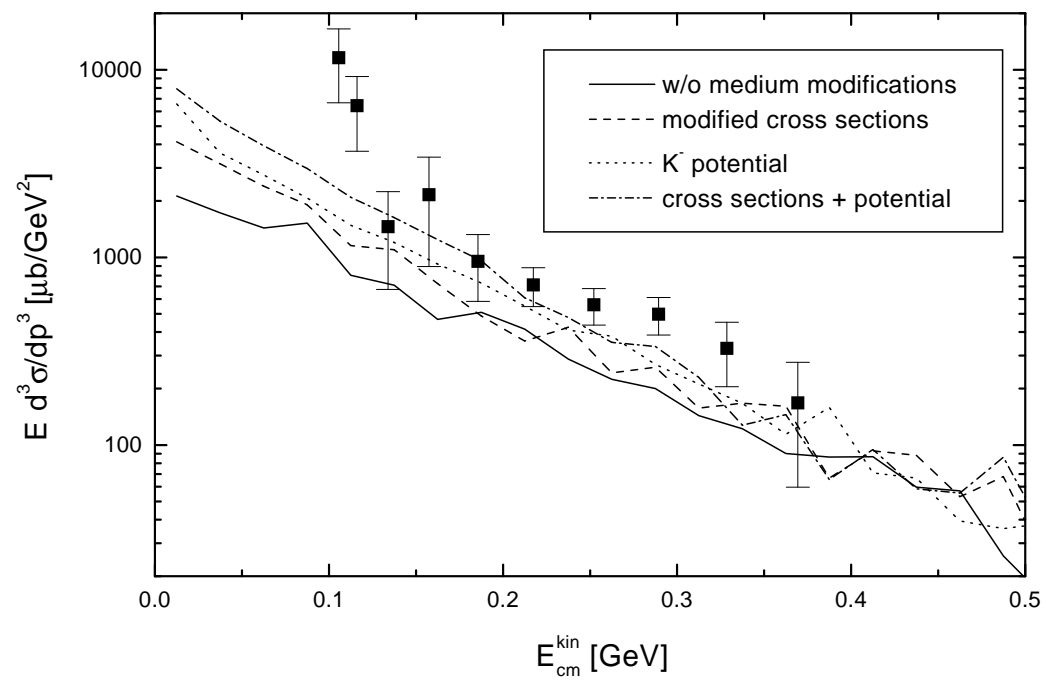

FIG. 12. Inclusive invariant $K^{-}$production cross section for $\mathrm{Ni}+\mathrm{Ni}$ collisions at $1.8 \mathrm{AGeV}$. Note that in the theoretical calculations the experimental acceptance cut is not applied. The experimental data are taken from Ref. [13]. 


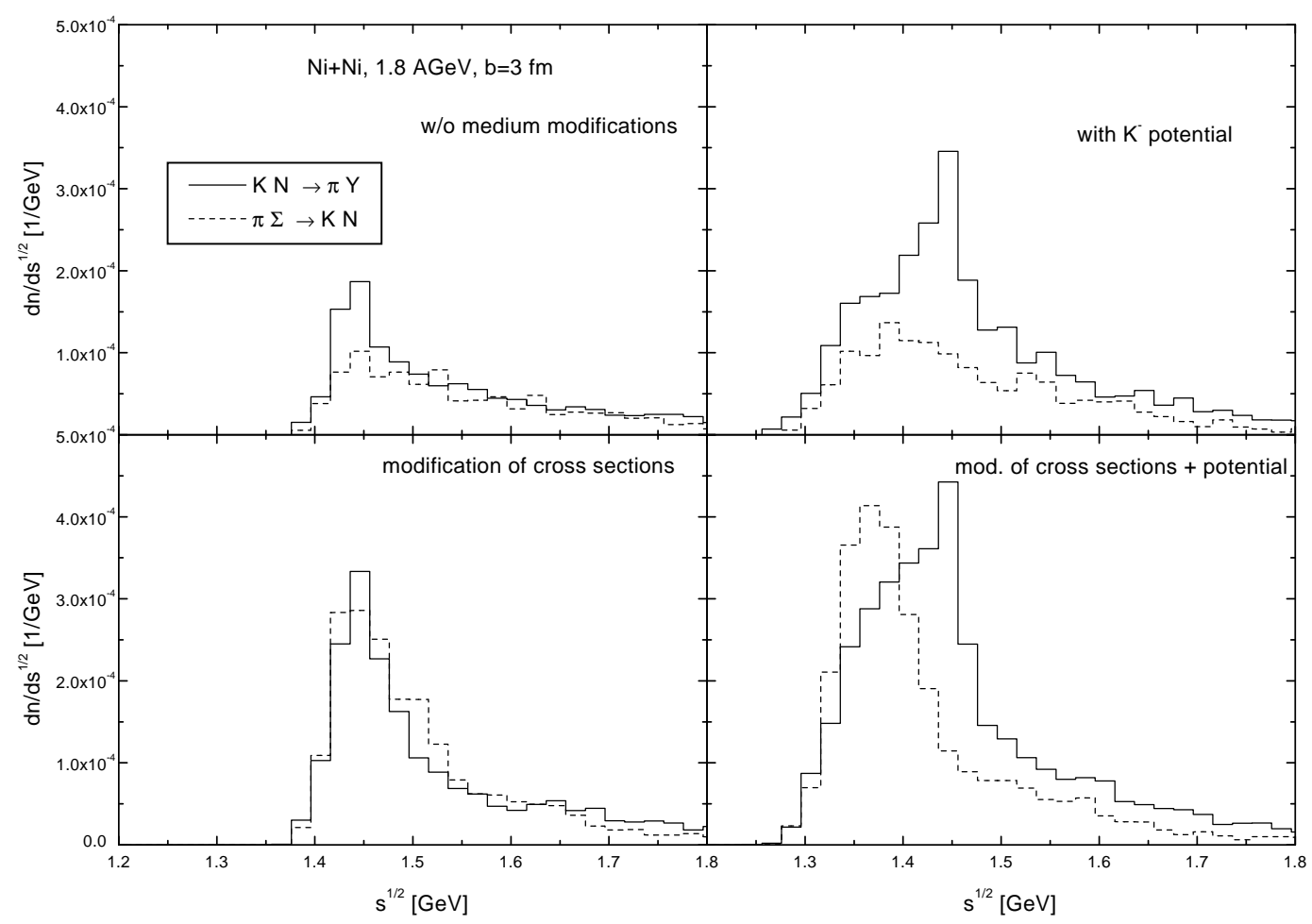

FIG. 13. Time integrated numbers of $\pi \Sigma \rightarrow \bar{K} N$ and $\bar{K} N \rightarrow \pi Y$ collisions as function of $\sqrt{s}$ for different medium modifications. The numbers were obtained from a calculation of a $\mathrm{Ni}+\mathrm{Ni}$ collision at $1.8 \mathrm{AGeV}$ and $b=3 \mathrm{fm}$.

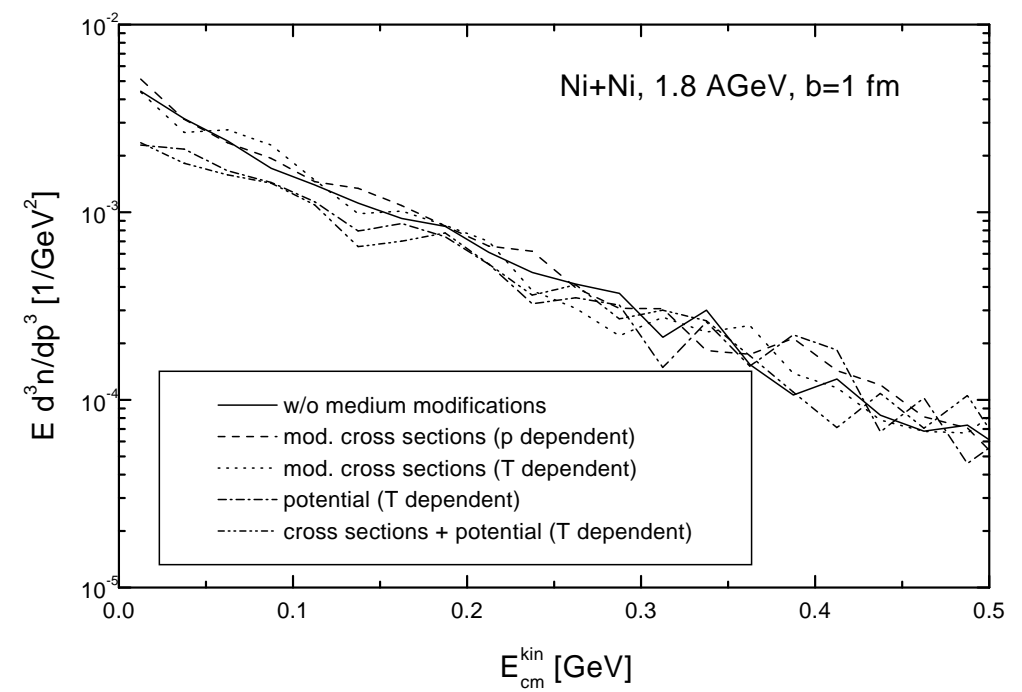

FIG. 14. Effects of momentum or temperature dependent in-medium modifications on the $K^{-}$ spectrum in a $\mathrm{Ni}+\mathrm{Ni}$ collision at $1.8 \mathrm{AGeV}$ and $b=1 \mathrm{fm}$. Fluctuations are caused by low numerical statistics. 


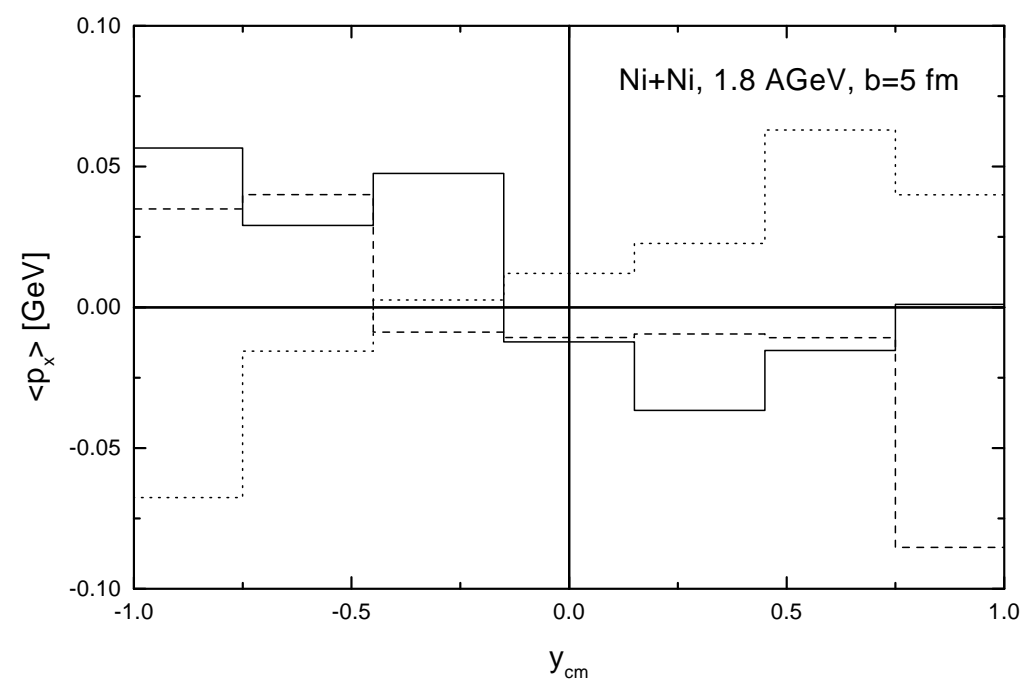

FIG. 15. Effects of in-medium modifications on transverse $K^{-}$flow in a $\mathrm{Ni}+\mathrm{Ni}$ collision at 1.8 $\mathrm{AGeV}$ and $b=3 \mathrm{fm}$ : Without in-medium modifications (solid line), with modified cross sections for $\pi Y \leftrightarrow \bar{K} N$ at $p=T=0$ (dashed line), with the $\bar{K}$-potential at $p=T=0$ (dotted line).

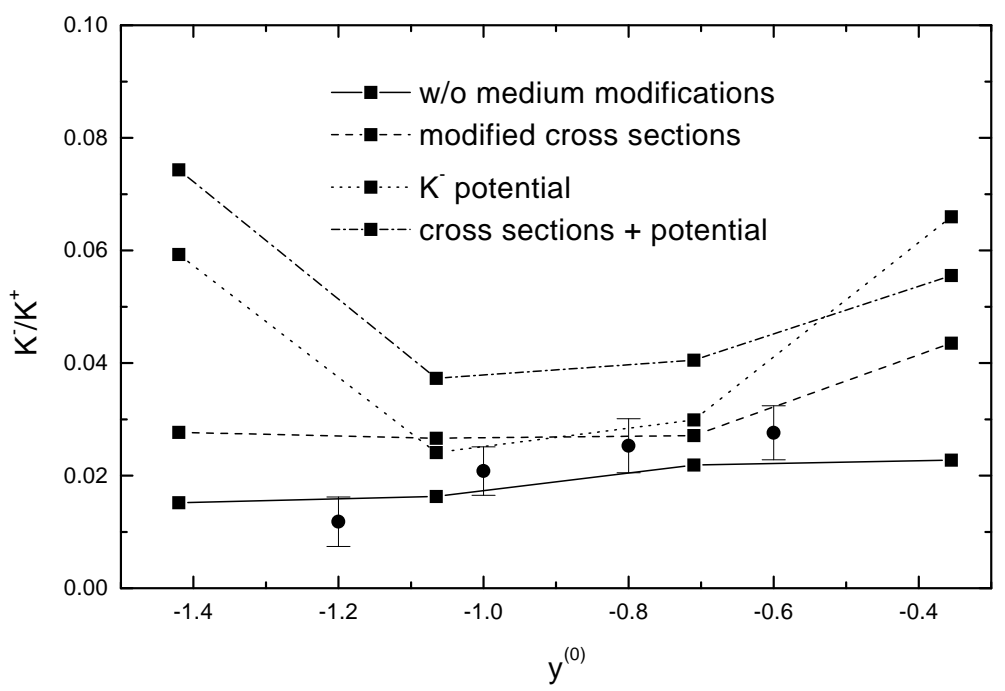

FIG. 16. Effects of in-medium modifications on the $K^{-} / K^{+}$ratio in $\mathrm{Ru}+\mathrm{Ru}$ collisions at 1.69 $\mathrm{AGeV}$ for $b<4 \mathrm{fm}$ as function of normalized rapidity $\left(y^{(0)}=y_{\text {beam }} / y_{C M S}-1\right)$ : Without in-medium modifications (solid line), with modified cross sections for $\pi Y \leftrightarrow \bar{K} N$ at $p=T=0$ (dashed line), with the $\bar{K}$-potential at $p=T=0$ (dotted line), with modified cross sections and potential (dash-dotted line). The experimental data are taken from Ref. [36]. 